\title{
Widespread Purple Plaques
}

Amena Raja Alkeswani, MD; James Robert Duncan, MD; Peter G. Pavlidakey, MD; Patricia Mercado, MD

Eligible for 1 MOC SA Credit From the ABD

This Photo Challenge in our print edition is eligible for 1 self-assessment credit for Maintenance of Certification from the American Board of Dermatology (ABD). After completing this activity, diplomates can visit the ABD website (http://www.abderm.org) to self-report the credits under the activity title "Cutis Photo Challenge." You may report the credit after each activity is completed or after accumulating multiple credits.
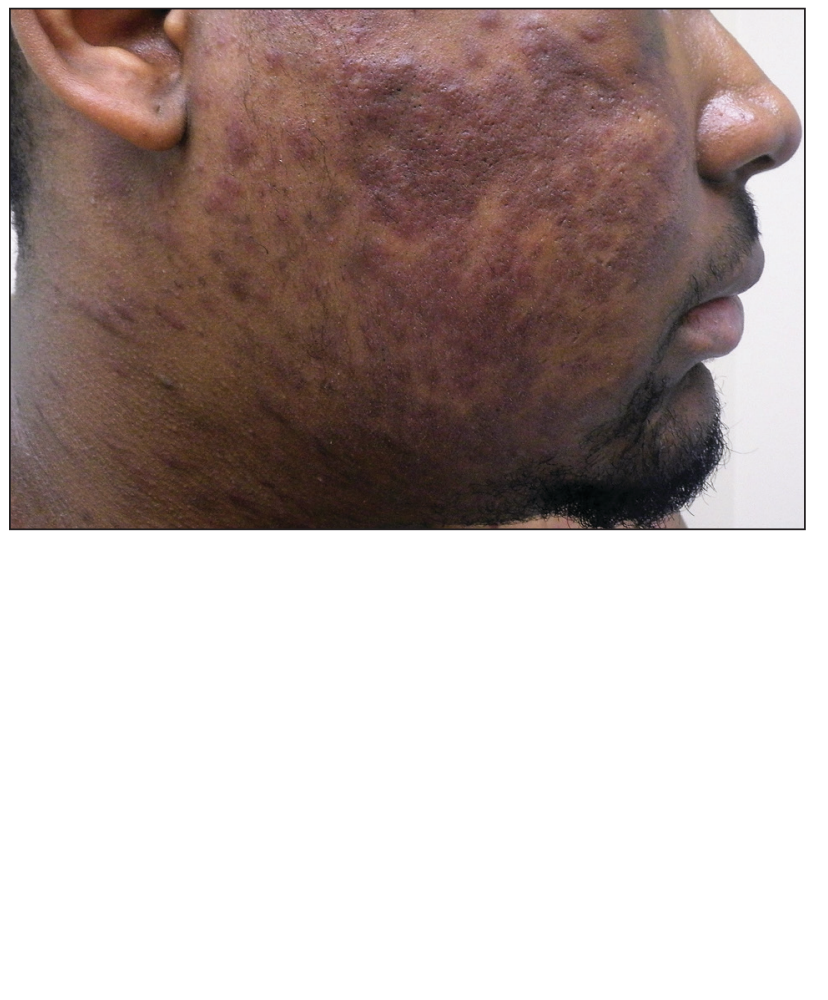

A 24-year-old Black man presented for evaluation of an asymptomatic, rash on the face, chest, back, and arms that had been progressively spreading over the course of 3 months. He had some swelling of the lips prior to the onset of the rash and was prescribed prednisone $10 \mathrm{mg}$ daily by an outside physician. He had no known medical problems and was taking no medications. Physical examination revealed numerous violaceous plaques scattered symmetrically on the trunk, arms, legs, and face. His family history was negative for autoimmune disease, and a review of systems was unremarkable. He denied any recent sexual contacts.

\section{WHAT'S YOUR DIAGNOSIS?}
a. Kaposi sarcoma
b. lichen planus pigmentosus
c. psoriasis
d. sarcoidosis
e. secondary syphilis 


\section{THE DIAGNOSIS: Kaposi Sarcoma}

O $\mathrm{n}$ initial presentation, the differential diagnosis included secondary syphilis, Kaposi sarcoma (KS), lichen planus pigmentosus, sarcoidosis, and psoriasis. A laboratory workup was ordered, which included complete blood cell count, comprehensive metabolic panel, antinuclear antibodies, anti-Ro/Sjögren syndrome antigen $\mathrm{A}$ and anti-La/Sjögren syndrome antigen $\mathrm{B}$ autoantibodies, angiotensin-converting enzyme, rapid plasma reagin, and human immunodeficiency virus (HIV) antibodies. A 4-mm punch biopsy of the rash also was performed from the right upper back. Histology revealed a vascular proliferation that was diffusely positive for human herpesvirus 8 (HHV-8)(Figure 1). The patient was informed of the diagnosis, at which time he revealed he had a history of homosexual relationships, with his last sexual contact being more than
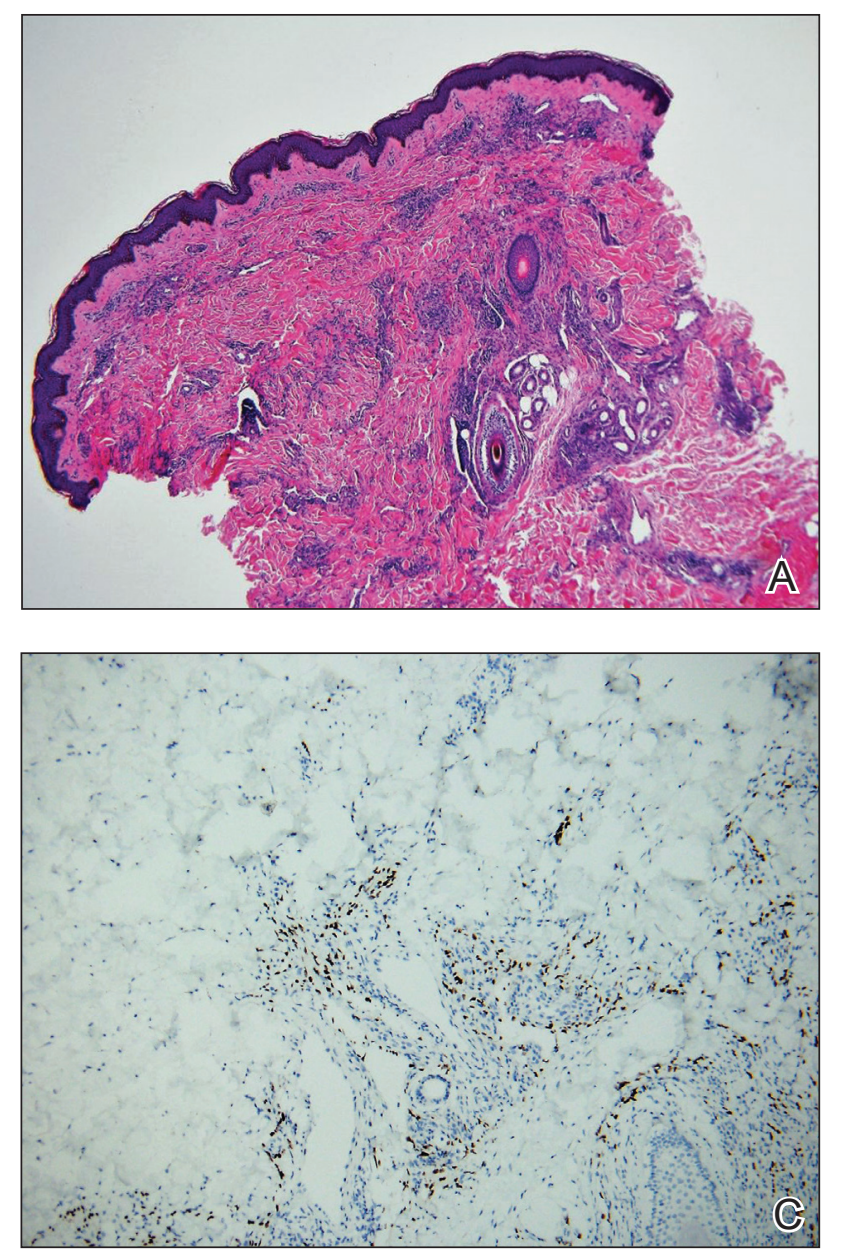

1 year prior to presentation. The laboratory workup confirmed a diagnosis of HIV, and the remainder of the tests were unremarkable.

He was referred to our university's HIV clinic where he was started on highly active antiretroviral therapy (HAART). His facial swelling worsened, leading to hospital admission. Computed tomography (CT) of the chest, abdomen, and pelvis showed diffuse lymphadenopathy and lung nodules concerning for visceral involvement of KS. Hematology and oncology was consulted for further evaluation, and he was treated with 6 cycles of doxorubicin $20 \mathrm{mg} / \mathrm{m}^{2}$, which led to resolution of the lung nodules on CT and improvement of the rash burden. He was then started on alitretinoin gel $0.1 \%$ twice daily, which led to continued slow improvement (Figure 2).
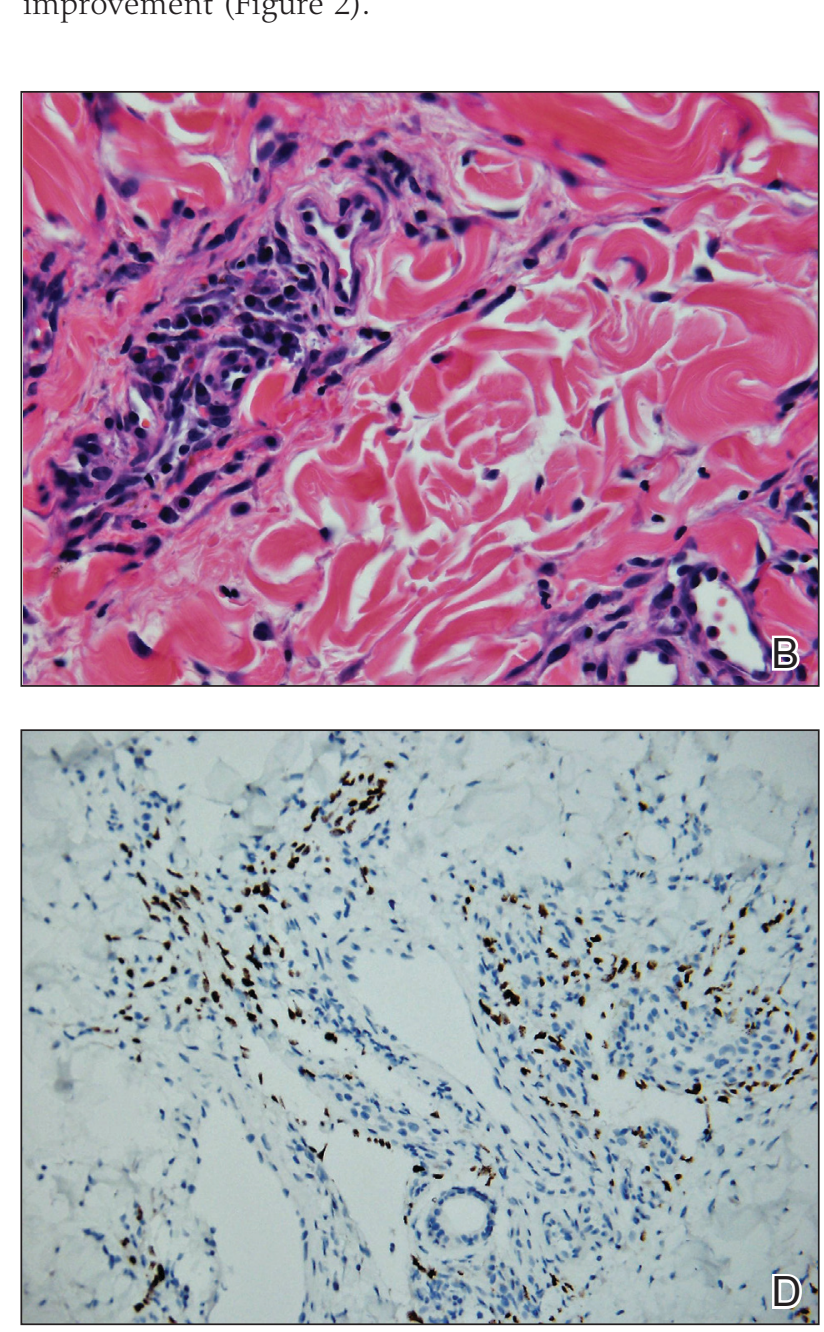

FIGURE 1. A and B, Histopathology showed a proliferation of endothelial cells forming vascular spaces infiltrating through collagen (H\&E, original magnifications $\times 10$ and $\times 40$ ). $C$ and $D$, A human herpesvirus 8 immunostain was positive within the endothelial cells (original magnifications $\times 10$ and $\times 40)$. 

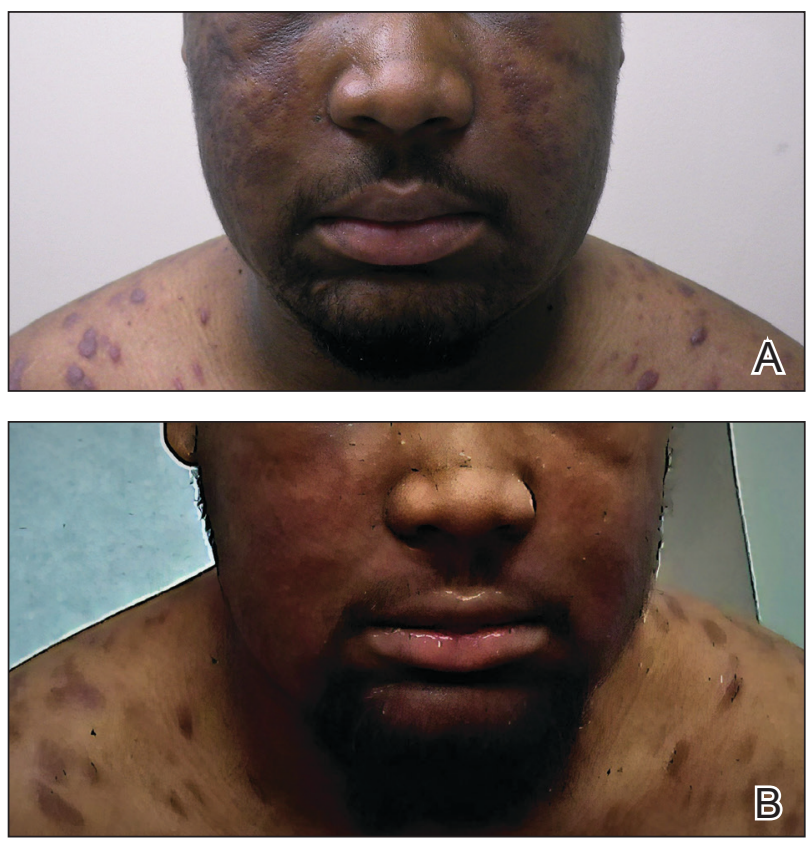

FIGURE 2. A and B, Widespread purple plaques at presentation and following treatment with highly active antiretroviral therapy, doxorubicin, and topical alitretinoin.

Kaposi sarcoma is a vascular neoplasm that occurs from infection with HHV-8. It typically presents as painless, reddish to violaceous macules or patches involving the skin and mucosa that often progress to plaques or nodules with possible visceral involvement. Kaposi sarcoma is classified into 4 subtypes based on epidemiology and clinical presentation: classic, endemic, iatrogenic, and AIDS associated. ${ }^{1,2}$

Classic KS primarily affects elderly males of Mediterranean or Eastern European descent, with a mean age of 64.1 years and a male to female ratio of 3 to 1 . It has an indolent course and a strong predilection for the skin of the lower extremities. The endemic form occurs mainly in Africa and has a more aggressive course, especially the lymphadenopathic type that affects children younger than 10 years. ${ }^{3}$ Iatrogenic KS develops in immunosuppressed patients, such as transplant recipients, and may regress if the immunosuppressive agent is stopped. ${ }^{1}$ Kaposi sarcoma is an AIDS-defining illness and is the most common malignancy in AIDS patients. It is strongly associated with a low CD4 count, which accounts for the notable decline in its incidence after the widespread introduction of HAART. ${ }^{1}$ Among HIV patients, KS has the highest incidence in men who have sex with men. This population has a higher seroprevalence of HHV-8, which suggests possible sexual transmission of HHV-8. AIDS-associated KS most commonly involves the lower extremities, face, and oral mucosa. It may have visceral involvement, particularly of the gastrointestinal and respiratory systems, which carries a poor prognosis. ${ }^{4,5}$

Approximately $40 \%$ of patients presenting with KS have gastrointestinal tract involvement. ${ }^{6}$ Of these patients, up to $80 \%$ are asymptomatic, with diagnosis usually being made on endoscopy. ${ }^{7}$ In contrast, pulmonary $\mathrm{KS}$ is less common and typically is symptomatic. It can involve the lung parenchyma, airways, or pleura and is diagnosed by chest radiography or CT scans. Glucocorticoid therapy is a known trigger for pulmonary KS exacerbation. ${ }^{8}$

All 4 subtypes share the same histopathologic findings consisting of spindled endothelial cell proliferation, inflammation, and angiogenesis. Immunohistochemistry reveals tumor cells that are CD34 and CD31 positive but are factor VIII negative. Staining for HHV-8 antigen is used to confirm the diagnosis. The inflammatory infiltrate predominantly is lymphocytic with scattered plasma cells. ${ }^{9}$

The laboratory results and histopathologic findings clearly indicated a diagnosis of KS in our patient. Other entities in the clinical differential would have shown notably different histopathologic findings and laboratory results. Lichen planus pigmentosus displays a lichenoid infiltrate and pigment dropout on histology. Histologic findings of psoriasis include psoriasiform acanthosis, dilated vessels in the dermal papillae, thinning of suprapapillary plates, and neutrophilic microabscesses. Sarcoidosis would demonstrate naked granulomas on histopathology. Syphilis displays variable but often psoriasiform or lichenoid findings on histology, and a positive rapid plasma reagin also would be noted.

First-line treatment of AIDS-related KS is HAART. For patients with severe and rapidly progressive KS or with visceral involvement, cytotoxic chemotherapy with doxorubicin or taxanes often is required. Additional therapies include radiotherapy, topical alitretinoin, and cryotherapy. ${ }^{1,10}$

\section{REFERENCES}

1. Schneider JW, Dittmer DP. Diagnosis and treatment of Kaposi sarcoma. Am J Clin Dermatol. 2017;18:529-539.

2. Schwartz RA, Micali G, Nasca MR, et al. Kaposi sarcoma: a continuing conundrum. J Am Acad Dermatol. 2008;59:179-206; quiz 207-208.

3. Mohanna S, MacoV, Bravo F, et al. Epidemiology and clinical characteristics of classic Kaposi's sarcoma, seroprevalence, and variants of human herpesvirus 8 in South America: a critical review of an old disease. Int J Infect Dis. 2005;9:239-250.

4. Beral V, Peterman TA, Berkelman RL, et al. Kaposi's sarcoma among persons with AIDS: a sexually transmitted infection? Lancet. 1990;335:123-128.

5. Smith NA, Sabin CA, Gopal R, et al. Serologic evidence of human herpesvirus 8 transmission by homosexual but not heterosexual sex. J Infect Dis. 1999;180:600-606.

6. Arora M, Goldberg EM. Kaposi sarcoma involving the gastrointestinal tract. Gastroenterol Hepatol (NY). 2010;6:459-462.

7. Parente F, Cernuschi M, Orlando G, et al. Kaposi's sarcoma and AIDS: frequency of gastrointestinal involvement and its effect on survival. a prospective study in a heterogeneous population. Scand J Gastroenterol. 1991;26:1007-1012.

8. Gasparetto TD, Marchiori E, Lourenco S, et al. Pulmonary involvement in Kaposi sarcoma: correlation between imaging and pathology. Orphanet J Rare Dis. 2009;4:18.

9. Radu O, Pantanowitz L. Kaposi sarcoma. Arch Pathol Lab Med. 2013;137:289-294.

10. Regnier-Rosencher E, Guillot B, Dupin N. Treatments for classic Kaposi sarcoma: a systematic review of the literature. J Am Acad Dermatol. 2013;68:313-331. 